\title{
Analysis of the generators of epileptic activity in early-onset childhood benign occipital lobe epilepsy
}

\author{
Alberto J.R. Leal ${ }^{\mathrm{a}, \mathrm{b}, *}$, Sofia Nunes ${ }^{\mathrm{c}}$, Ana I. Dias ${ }^{\mathrm{b}}$, José P. Vieira ${ }^{\mathrm{b}}$, \\ Ana Moreira ${ }^{\mathrm{b}}$, Eulália Calado ${ }^{\mathrm{b}}$ \\ ${ }^{a}$ Department of Neurophysiology, Hospital Júlio de Matos, Av. do Brasil nr 53, 1749-002 Lisbon, Portugal \\ ${ }^{\mathrm{b}}$ Department of Pediatric Neurology, Hospital Dona Estefânia, Lisbon, Portugal \\ ${ }^{\mathrm{c}}$ Department of Pediatrics, Hospital Fernando Fonseca, Amadora, Portugal
}

Accepted 7 February 2007

Available online 29 March 2007

\begin{abstract}
Objective: The Panayiotopoulos type of idiopathic occipital epilepsy has peculiar and easily recognizable ictal symptoms, which are associated with complex and variable spike activity over the posterior scalp areas. These characteristics of spikes have prevented localization of the particular brain regions originating clinical manifestations. We studied spike activity in this epilepsy to determine their brain generators.

Methods: The EEG of 5 patients (ages 7-9) was recorded, spikes were submitted to blind decomposition in independent components (ICs) and those to source analysis (sLORETA), revealing the spike generators. Coherence analysis evaluated the dynamics of the components.

Results: Several ICs were recovered for posterior spikes in contrast to central spikes which originated a single one. Coherence analysis supports a model with epileptic activity originating near lateral occipital area and spreading to cortical temporal or parietal areas.

Conclusions: Posterior spikes demonstrate rapid spread of epileptic activity to nearby lobes, starting in the lateral occipital area. In contrast, central spikes remain localized in the rolandic fissure.

Significance: Rapid spread of posterior epileptic activity in the Panayitopoulos type of occipital lobe epilepsy is responsible for the variable and poorly localized spike EEG. The lateral occipital cortex is the primary generator of the epileptic activity.

(C) 2007 International Federation of Clinical Neurophysiology. Published by Elsevier Ireland Ltd. All rights reserved.
\end{abstract}

Keywords: Epilepsy; Panayiotopoulos syndrome; Occipital lobe; sLORETA; Idiopathic; ICA

\section{Introduction}

The Panayiotopoulos type of idiopathic occipital lobe epilepy (IOLE) has been a focus of research in epilepsy in recent years (Panayiotopoulos, 2002) but major issues still remain unsolved such as the particular brain area originating the seizure clinical symptoms and also the significance of the variability of posterior spike activity (Ohtsu et al., 2003). In this respect there is a sharp contrast with the

\footnotetext{
${ }^{*}$ Corresponding author. Present address: Department of Neurophysiology, Hospital Júlio de Matos, Av. do Brasil nr 53, 1749-002 Lisbon, Portugal. Tel.: +351 969851734; fax: +351 217819809 .

E-mail address: a.leal@netcabo.pt (A.J.R. Leal).
}

syndrome of idiopathic epilepsy with rolandic spikes, which is associated with a consistent neurophysiological picture, pointing to the brain area of seizure onset in the rolandic fissure (Wolff et al., 2005).

The source analysis methods in EEG (Yoshinaga et al., 2005, 2006) and MEG (Kanazawa et al., 2006), using the equivalent current dipole, suggest origin of the epileptic activity near the calcarine sulcus and parieto-occipital areas. These results do not provide a good explanation to the typical ictal clinical manifestations and also fail to explain why the visual symptoms are so rare in the syndrome.

In this work we perform a neurophysiological study of posterior EEG spikes in cases of the Payionotopoulos type 
Table 1

Clinical data

\begin{tabular}{|c|c|c|c|c|c|}
\hline & Patient 1 & Patient 2 & Patient 3 & Patient 4 & Patient 5 \\
\hline Age of onset (Y) & 6 & 3 & 6 & 2 & 4 \\
\hline Development & Normal & Normal & Normal & Normal & Cerebral palsy \\
\hline \multicolumn{6}{|l|}{ Ictal symptoms } \\
\hline Vomiting & no & Yes & Yes & Yes & Yes \\
\hline Eye deviation & Yes & No & Yes & Yes & No \\
\hline Decreased reactivity & Yes & No & No & Yes & Yes \\
\hline Other & & Nistagmus, sialorrea & Pallor & & Decreased tonus \\
\hline Seizure duration & & $>20 \mathrm{~min}$ & $5-10 \mathrm{~min}$ & $>15 \min$ & $>20 \mathrm{~min}$ \\
\hline Number of seizures & 1 & 4 & 2 & 4 & 3 \\
\hline
\end{tabular}

of IOLE and a comparison is made with rolandic spikes also present in some of these patients. Our main goal is to determine the usefulness of the interictal EEG analysis to highlight the localization of the primary epileptic foci in this syndrome.

\section{Methods and subjects}

Five patients with the diagnosis of early onset IOLE (Panayiotopoulos type) were selected from the epilepsy outpatient clinic of Hospital Dona Estefânia. All demonstrated clinical (Table 1) and neurophysiological (Table 2) data compatible with the ILAE criteria for the diagnosis (Covanis et al., 2005).

Patients were studied in two moments, with a temporal interval of 3 years. In the early study, a special cap with 30 electrodes (10-20 plus $\mathrm{Fp}_{\mathrm{z}}, \mathrm{P}_{9 / 10}, \mathrm{PO}_{3 / 4}, \mathrm{PO}_{7 / 8}, \mathrm{O}_{z}, \mathrm{O}_{9 / 10}$ and $\mathrm{PO}_{\mathrm{z}}$ ) was used to perform a sleep study with 1-h duration. For the repeated study a 24-h ambulatory EEG was performed with 30 electrodes individually glued to the scalp (10-20 plus $\mathrm{F}_{9 / 10}, \mathrm{~T}_{9 / 10}, \mathrm{P}_{9 / 10}, \mathrm{PO}_{3 / 4}, \mathrm{O}_{9 / 10}$ and $\mathrm{O}_{z}$ ). The electrodes were photographed in several planes and their position in the scalp reproduced in a $3 \mathrm{D}$ rendering of a standard average brain. The sampling rate for both recordings was $256 \mathrm{~Hz}$ with high- and low-pass filters at 1 and $70 \mathrm{~Hz}$, respectively.

The EEG recordings were visually reviewed by a clinical neurophysiologist (AL) and the peak of individual spikes marked. Because some of the patients had spikes with different topographies, we selected a minimum of 40 spikes (average of $91 \pm 26$ ) for each spike type. This proved possible because all patients had abundant spikes during the sleep period.

The EEG spikes with no artifacts associated were cut in epochs ( -100 to $300 \mathrm{~ms}$ ) around the voltage peak, using the Scan 4.3.1 (Neuroscan, El Paso) software. Data were exported to the EEGLAB 4.515 package (Delorme and Makeig, 2004) for decomposition of the raw spike groups in independent components, using the Infomax algorithm (Bell and Sejnowski, 1995) and methods detailed elsewhere (Leal et al., 2006). The spatial components with consistent activation at the time of spike peak were selected and submitted to cross-coherence analysis in order to obtain data on their temporal dynamics.

The independent component analysis (ICA) components were sorted by time of activation, providing information on the spreading patterns of the spike activity, and later submitted to source analysis to improve localization of the cortical areas involved.

Source analysis was done using the sLORETA software package (Pascual-Marqui, 2002) available at http://www. unizh.ch/keyinst/NewLORETA/LORETA01.htm. The maximum of the current density obtained at a given moment was taken as the source of the particular component, and because the spatial components recovered from

Table 2

Neurophysiological data

\begin{tabular}{|c|c|c|c|c|c|}
\hline & Patient 1 & Patient 2 & Patient 3 & Patient 4 & Patient 5 \\
\hline \multicolumn{6}{|l|}{ Early study } \\
\hline Age & 7 & 7 & 8 & 9 & 9 \\
\hline Background & Normal & Normal & Normal & Normal & Normal \\
\hline Interictal & $\begin{array}{l}\text { Left occipital } \\
\text { spikes }\end{array}$ & Parietal spikes & $\begin{array}{l}\text { Right central and } \\
\text { left occipital spikes }\end{array}$ & $\begin{array}{l}\text { Right central and } \\
\text { occipital spikes }\end{array}$ & $\begin{array}{l}\text { Left central and } \\
\text { occipital spikes }\end{array}$ \\
\hline Early generator & Left occipital & Parietal-occipital & Left occipital & Right occipital & Left occipital \\
\hline Spread areas & Left temporal & & Left temporal and parietal & Right Parietal & Left parietal \\
\hline \multicolumn{6}{|l|}{ Second study } \\
\hline Age & 10 & 10 & 11 & 11 & 12 \\
\hline Background & Normal & Normal & Normal & Normal & Normal \\
\hline Interictal & Left occipital spikes & Parietal spikes & Right central spikes & Right central and parietal spikes & Left central spikes \\
\hline Early generator & Left occipital & Parietal-occipital & & Right parietal & \\
\hline Spread areas & Left temporal & & & Right central & \\
\hline
\end{tabular}


the ICA decomposition are fixed, the source localization also remains fixed along time.

\section{Results}

Most patients (3/5) demonstrated two independent spike topographies, with maximum over posterior and central areas (Fig. 1). The latter demonstrated the typical features of rolandic spikes, with maximum amplitude in the central areas and a transverse dipolar type scalp potential, positive over the frontal areas. The posterior spikes had the features described in the literature for the Panayiotopoulos type of occipital lobe epilepsy (Ohtsu et al., 2003) (Fig. 1).

Because we were interested in evaluating the temporal stability of these interictal patterns, two studies were performed with an average separation of 3 years. When a comparison was made between the two evaluations, we found that for the three patients with central spikes in the initial study, those were also present in the second one and had a similar topography. The posterior spikes persisted in three out of five patients and disappeared in the remaining ones, despite the fact that a long recording session $(24 \mathrm{~h})$ was done in the second evaluation. In two out of three patients the topography of these spikes remained very similar in the two studies, but there was a shift toward the parietal lobes in the remaining one.

The averaged spikes demonstrated a dipolar type of scalp potential, which produced a clear focal maximum in the sLORETA source analysis method (Fig. 1).

Because we are interested in evaluating the dynamics of the interictal activity, the independent spikes of each topographic group were submitted to decomposition in independent components instead of averaging, reconstructing the signal as a linear combination of fixed spatial and variable temporal components (Makeig et al., 2004). The spatial components provide information on the localization of brain generators, while the temporal components provide the temporal sequence of activation. Furthermore the number of ICs with consistent activation time-locked to the spike peak provides information on the minimum number of intracranial generators to include in the model (MarcoPallarés et al., 2005).

The posterior spikes produced several ICs (Figs. 2-4), with distinct spatial and temporal characteristics. The analysis of the relative time of activation of their generators suggests that in these spikes different brain areas contribute

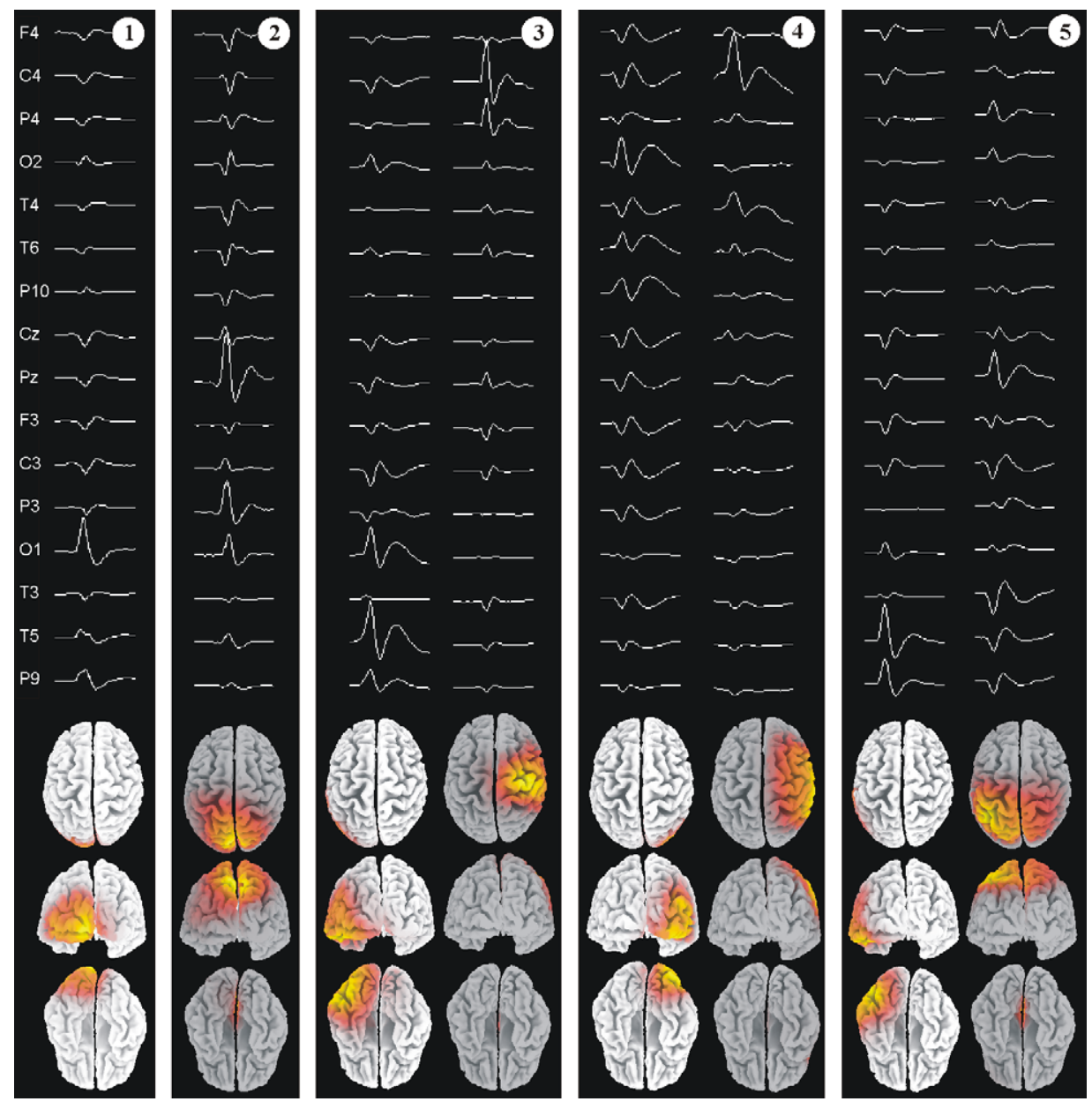

Fig. 1. Topographies of interictal spikes for the five patients. (Above) Sub-sample of the averaged spikes, demonstrating multifocal activity for patients 3 , 4 and 5, with a combination of rolandic and posterior spikes. (Below) Current source distribution at spike peak using the sLORETA algorithm constrained to the cortical volume, demonstrating single maxima of current density in the more superficial cortical areas. 

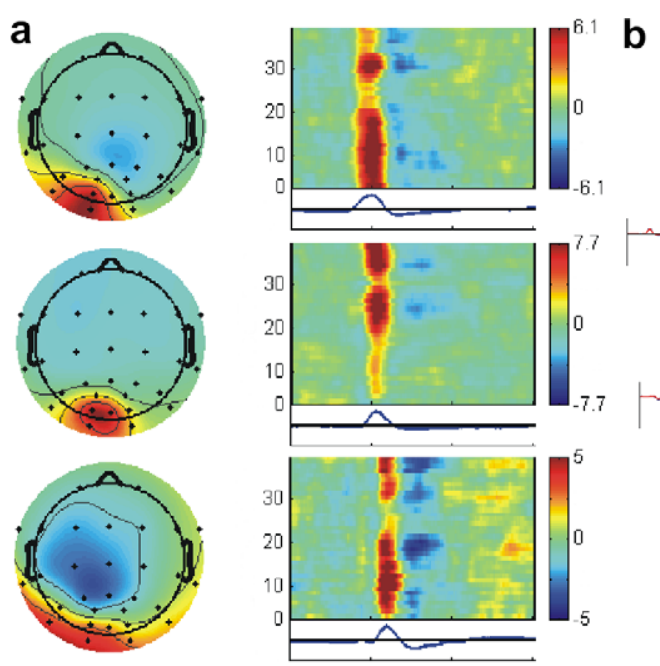

b
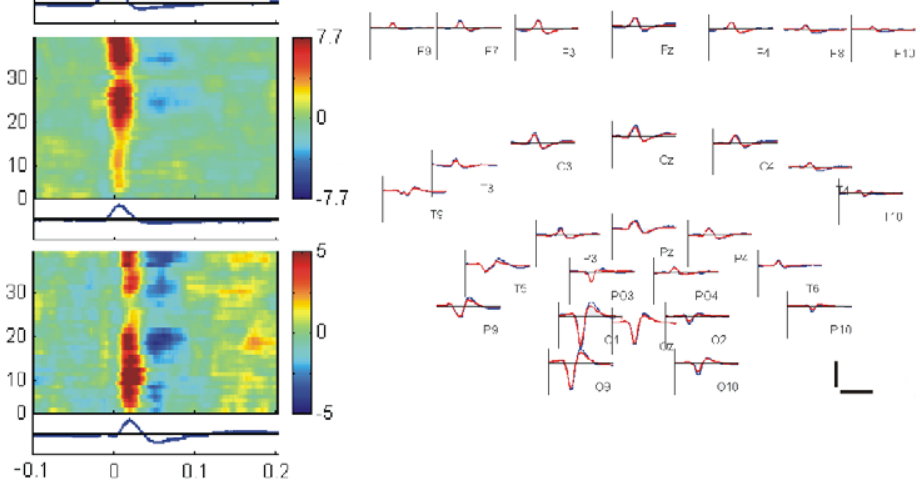

c

d
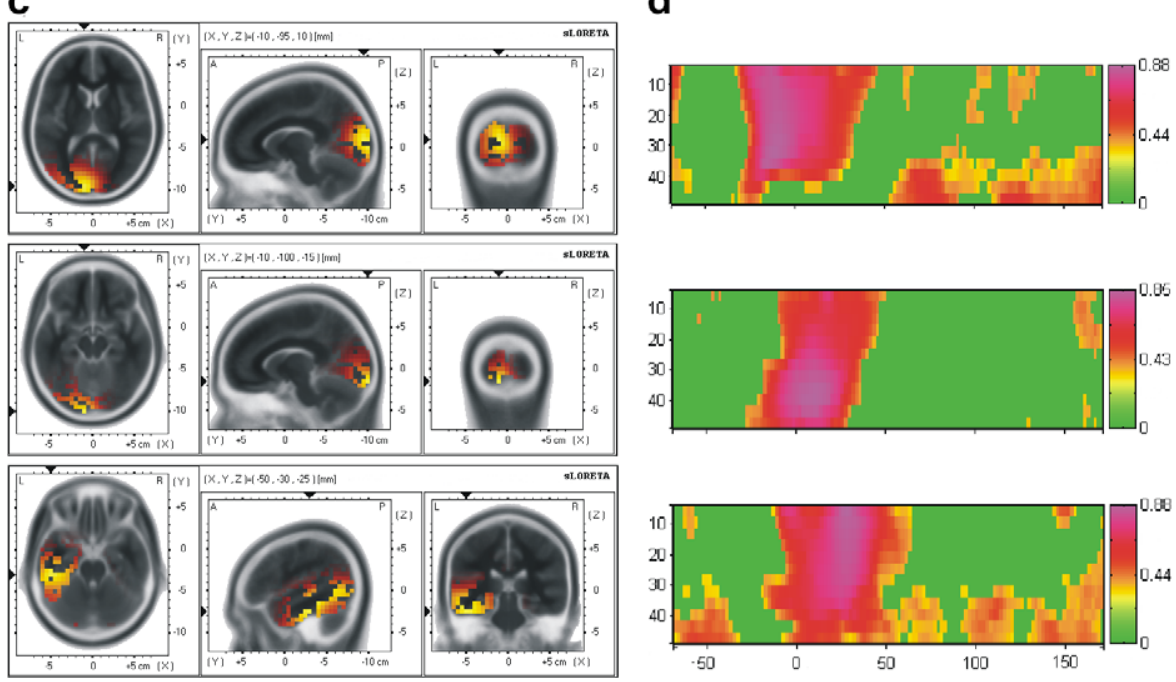

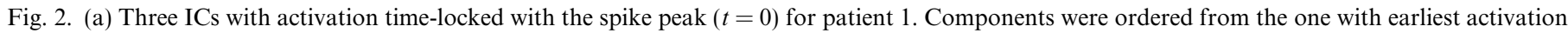

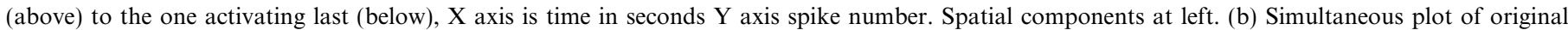

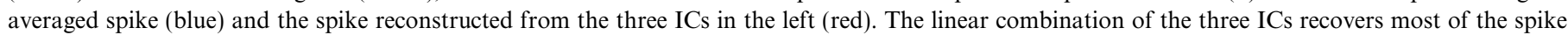

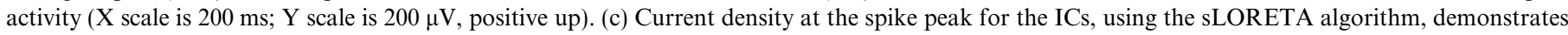

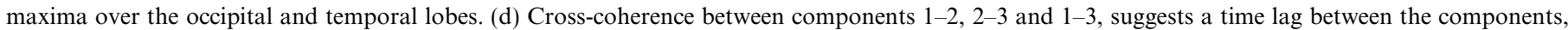

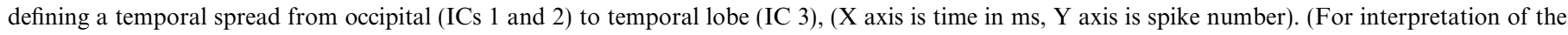
references in colour in this figure legend, the reader is referred to the web version of this article.)

to the potential in the scalp, and the consistent temporal shifts between them (Figs. 2 and 3) further suggest that this is due to spread of the epileptic activity to nearby areas, from a primary source in the polar or lateral occipital lobes (Fig. 4).

The central spikes produced only one or two close-by ICs for each patient, with a single maximum (Fig. 5). This suggests that no significant spread of epileptic activity takes place in these paroxysms. They also remained stable throughout time in three out of three patients.

\section{Discussion}

The analysis of the spatial and temporal dynamics of occipital and rolandic spikes in the Panayiotopoulos type of occipital lobe epilepsy reveals an important distinction: while rolandic spikes decompose in a single component with maximum over the central areas, suggesting a focal area of onset with little spread, the occipital spikes produce several ICs with distinct spatial distribution and times of activation, supporting the existence of significant spread of epileptic activity to neighboring areas from a point of origin in the superficial posterior and lateral occipital lobes.

The general characteristics of the clinical manifestations in our patients are typical of the ones described for the Panayiotopoulos type of childhood occipital lobe epilepsy (Panayiotopoulos, 1989), with autonomic ictal manifestations associated with decreased consciousness and eye deviation. The seizures lasted several minutes and were rare events (Table 1), which are also common features of the syndrome. Patient 5 had cerebral palsy but exhibited long lasting seizures with autonomic manifestations and 

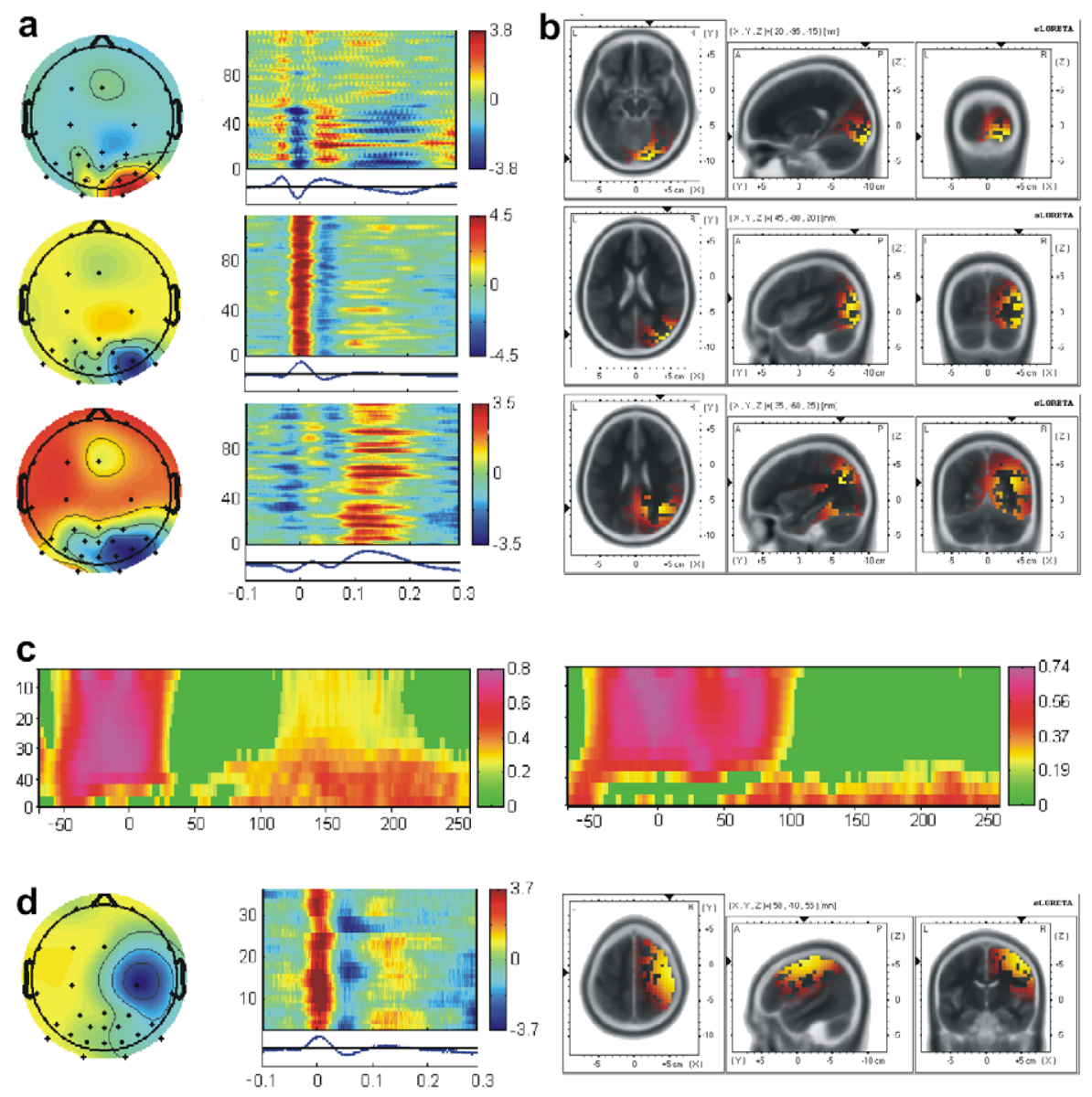

Fig. 3. (a) ICs time-locked with the spike peak for patient 4, ordered by sequential time of activation. (b) Current density maxima for the different ICs using the sLORETA solution at spike peak. (c) Cross-coherence between components 1-3 and 1-2. (d) IC for the central spike, with sLORETA solution compatible with a rolandic focus (legends as in Fig. 1).

decreased reactivity which fulfils the criteria for the syndrome, despite the static neurological deficit (Table 2).

The interictal EEGs demonstrated the typical highamplitude posterior spikes, which in three out of five cases was accompanied by independent rolandic spikes. The coexistence of these spike types was reported by Panayiotopoulos (2002) and has in fact motivated the suggestion that a continuum might exist between this syndrome and the Benign Epilepsy with Rolandic Spikes. In two of our patients the posterior spikes disappeared in the second study, while rolandic spikes persisted. This supports an age-dependent expression of the spike activity, with posterior spikes disappearing before the central ones.

The decomposition in independent components of the EEG time series is a proven and robust procedure to decrease the mixing of different brain generators in the scalp electrodes due to volume conductor effects (Makeig et al., 2004). In our patients this analysis suggests that for rolandic and posterior spikes, despite a similar surface morphology, very different number of brain generators are involved; while in the first case a single and focal generator explains well the surface morphology (Figs. $3 b$ and 5), for the latter spike type several focal generators are required
(Figs. 2, 3a and 4), suggesting the existence of significant volume conductor mixing in the scalp record of these spikes. The detailed analysis of the time of onset of the ICs demonstrates consistent time lags, which are more compatible with epileptic activity spread between the different areas than with a simultaneous onset. From an area of onset in the posterior and superficial occipital lobes different patterns of spreading can be defined and this may be related with different clinical manifestations. The reduced number of our patients precludes a robust analysis of this possibility, but several trends are apparent such as: persistent existence of interruption of consciousness in patients with temporal lobe spread (patients 1, 4 and 5); vomiting in patients with parietal lobe spread (patients 2, 3, 4 and 5 ); eye deviation in patients with lateral occipital lobe foci (patients 1, 3 and 4).

The source analysis method used to obtain the spatial localization of the independent generators belongs to the class of linear methods (Pascual-Marqui, 1999), where a large number of fixed dipoles contribute to the overall solution. Such methods are not so sensitive to errors produced by a wrong choice of the number of intracranial generators (Pascual-Marqui, 1999) and can incorporate 


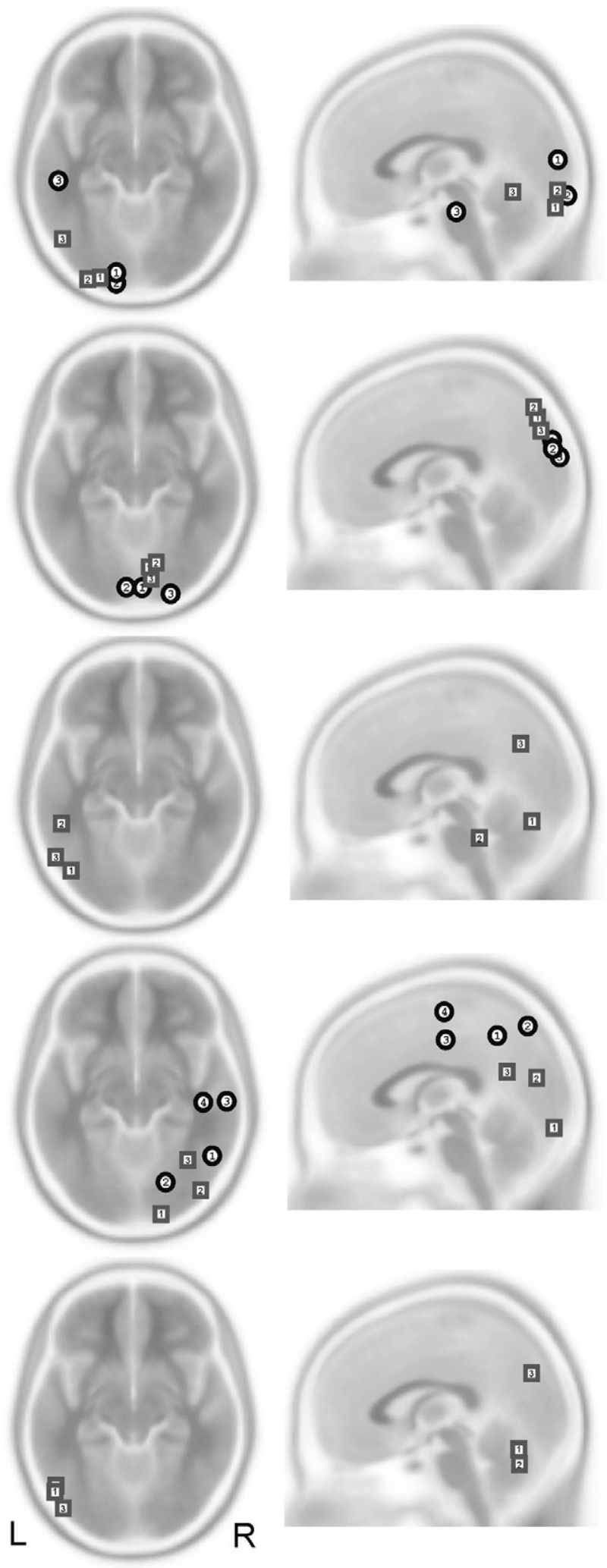

Fig. 4. Propagation patterns for the posterior spikes (patients 1 to 5, from above-down), demonstrating early components (1) in posterior or lateral occipital cortex, spreading to the temporal lobe (patients 1 and 3 ) or to the parietal lobe (patients 3, 4 and 5). Results for the first (squares) and second studies (circles), demonstrate overlap of results (patients 1 and 2), discrepant results (patient 4) or disappearance of posterior spikes (patients 3 and 5).
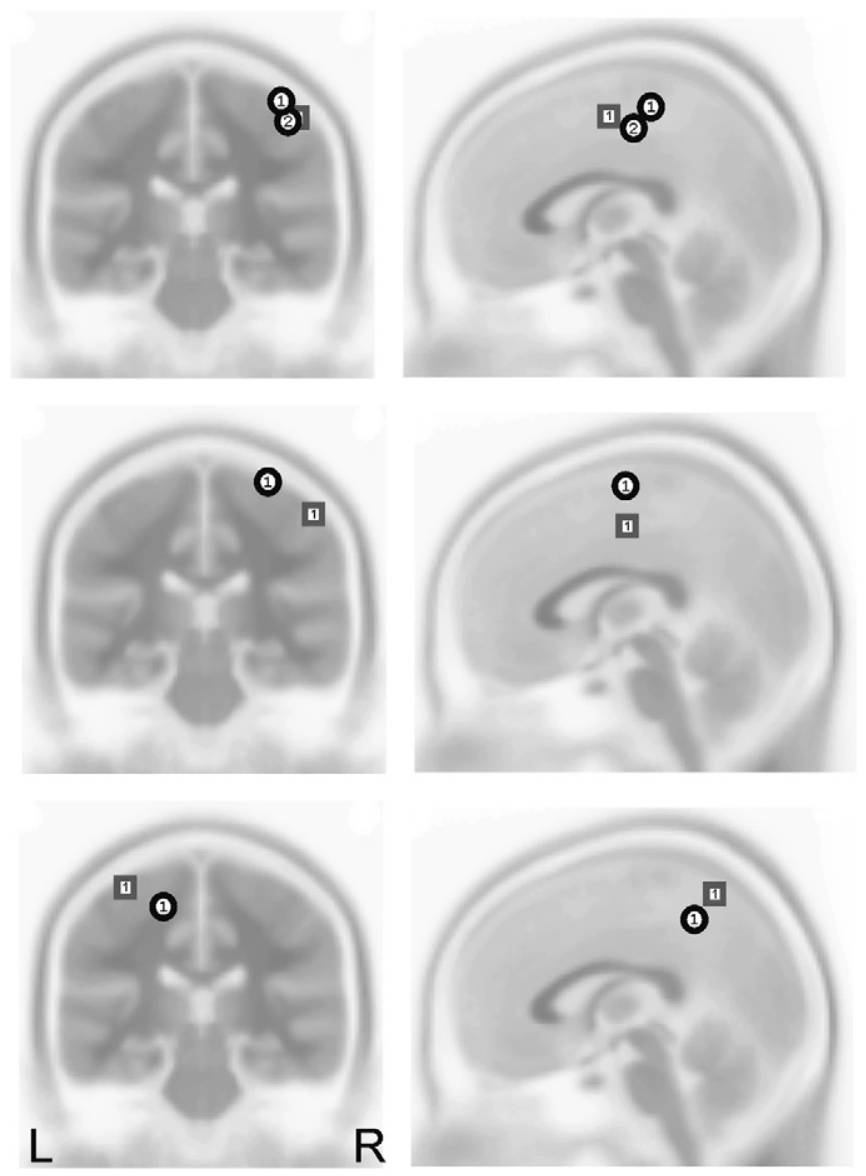

Fig. 5. Propagation patterns and temporal evolution of central spike generators for patients 3 (above), 4 (middle) and 5 (below). No significant spread is apparent, and the localization of the generators remained stable in the three-year interval of the two records (squares, first study; circles, second study).

easily anatomical constraints, which is a major advantage as compared to equivalent current dipole models. In our study the method was used to give an anatomical context to the spatial independent components, making more understandable their intracranial dynamics.

\section{Acknowledgements}

The authors wish to express their gratitude to Elisa Vilares, Adilia Seabra and Ana Beato for technical support.

\section{References}

Bell AJ, Sejnowski TJ. An information-maximization approach to blind separation and blind deconvolution. Neural Comput 1995;7:1129-59.

Covanis A, Ferrie CD, Koutroumanidis M, Oguni H, Panayiotopoulos CP. Panayotopoulos syndrome and Gastaut type idiopathic childhood occipital epilepsy. In Roger J, Bureau M, Dravet Ch, Genton P, Tassinari CA, Wolf P. Epileptic syndromes in infancy, childhood and adolescence. 2005; pp. 227-253: 4th ed, John Libbey.

Delorme A, Makeig S. EEGLAB: an open source toolbox for analysis of single-trial Eeg dynamics including independent components. J Neurosci Methods 2004;134(1):9-21. 
Kanazawa O, Tohyama J, Akasaka N, Kamimura T. A magnetoencephalographic study of patients with Panayiotopoulos syndrome. Epilepsia 2006;46(7):1106-13.

Leal AJ, Dias AI, Vieira JP. Analysis of the EEG dynamics of epileptic activity in gelastic seizures using decomposition in independent components. Clin Neurophysiol 2006;117:1595-601.

Makeig S, Debener S, Onton J, Delorme A. Mining event-related brain dynamics. Trends Cogn Sci 2004;8(5):204-10.

Marco-Pallarés J, Grau C, Ruffini G. Combined ICA-LORETA analysis of mismatch negativity. Neuroimage 2005;25:471-7.

Ohtsu M, Oguni H, Hayashi K, Funatsuka M, Imai K, Osawa M. EEG in children with early-onset benign occipital seizure susceptibility syndrome: Panayiotopoulos syndrome. Epilepsia 2003;44(3):435-42.

Panayiotopoulos CP. Benign childhood epilepsy with occipital paroxysms: a 15-year prospective study. Ann Neurol 1989;26:51-6.
Panayiotopoulos CP. Panayiotopoulos syndrome: a common and benign childhood epileptic syndrome. London: John Libbey; 2002.

Pascual-Marqui RD. Review of methods for solving the EEG inverse problem. Int J Bioelectromagnetics 1999;1:75-86.

Pascual-Marqui RD. Standardized low-resolution brain electromagnetic tomography (sLORETA): technical details. Methods Find Exp Clin Pharmacol 2002;24D:5-12.

Wolff M, Weiskopf N, Serra E, Preissl H, Birbaumer N, Kraegeloh-Mann. Benign partial epilepsy in childhood: selective cognitive deficits are related to the location of focal spikes determined by combined EEG/ MEG. Epilepsia 2005;46(10):1661-7.

Yoshinaga H, Koutroumanidis M, Shirasawa A, Kikumoto K, Ohtsuja Y, Oka E. Dipole analysis in Panayiotopoulos syndrome. Brain Dev 2005;27:46-52.

Yoshinaga H, Koutroumanidis M, Kobayashi K, Shirasawa A, Kikumoto $\mathrm{K}$, Inoue $\mathrm{T}$, et al. EEG dipole characteristics in Panayotopoulos syndrome. Epilepsia 2006;47(4):781-7. 